\title{
Reflets
}

Revue d'intervention sociale et communautaire

\section{Le militantisme en milieu rural et minoritaire francophone}

\section{Anne Jutras}

Volume 23, numéro 2, automne 2017

URI : https://id.erudit.org/iderudit/1043308ar

DOI : https://doi.org/10.7202/1043308ar

Aller au sommaire du numéro

Éditeur(s)

Reflets, Revue d'intervention sociale et communautaire

ISSN

1203-4576 (imprimé)

1712-8498 (numérique)

Découvrir la revue

Citer cet article

Jutras, A. (2017). Le militantisme en milieu rural et minoritaire francophone. Reflets, 23(2), 218-224. https://doi.org/10.7202/1043308ar d'utilisation que vous pouvez consulter en ligne.

https://apropos.erudit.org/fr/usagers/politique-dutilisation/ 


\title{
Le militantisme en milieu rural et minoritaire francophone
}

\author{
Anne Jutras, directrice générale \\ Centre Novas - CALACS francophone de Prescott-Russell
}

\section{Introduction}

Qu'est-ce que le militantisme, et comment cela s'applique-t-il en milieu rural? Certes, cela représente des défis intéressants et, lorsqu'on y ajoute la composante francophone en milieu minoritaire, il faut faire preuve de créativité! Voyez par vous-même!

Dans le texte qui suit, nous cherchons à vous présenter comment un CALACS (Centre d'aide et de lutte contre les agressions à caractère sexuel) francophone en région rurale ontarienne tente d'être militant dans sa communauté. Nous n'avons pas la prétention d'avoir toutes les réponses et nous faisons encore des essais-erreurs. La recette n'est pas encore parfaite, mais nous avons atteint un certain niveau de succès. Nous relatons donc ici notre expérience.

Le Centre Novas - CALACS francophone de Prescott-Russell, un organisme à but non lucratif, dessert les femmes francophones victimes et survivantes d'agression à caractère sexuel. Prescott-Russell est une région agricole qui s'étend sur $2002 \mathrm{~km}$ carrés et où les distances entre communautés et voisins sont grandes. Cette communauté est composée de deux comtés, soit Prescott et Russell, qui regroupent huit municipalités locales.

Le Centre Novas a ouvert ses portes en 2006. Il s'agit du seul centre pour femmes francophones spécialisé en matière d'agression à caractère sexuel dans cette région. Actif dans l'éducation, la prévention et la sensibilisation auprès de la population, le Centre Novas - CALACS francophone de Prescott-Russell offre une gamme de services d'aide et de soutien aux femmes en matière d'agression à caractère sexuel dans le but d'éliminer la violence faite aux femmes. Sa vision est de vivre dans une société égalitaire sans violence.

Le mandat du Centre inclut la défense des droits des femmes et la prévention de la violence faite aux femmes. Ses travaux s'appuient sur l'analyse, l'approche et l'intervention féministe. ${ }^{2}$ 


\section{Le militantisme, c'est quoi?}

De façon générale, le militantisme est une activité de lutte pour une cause. C'est bien, mais, à notre sens, c'est beaucoup plus que cela. Dans le militantisme, il faut avoir un sens d'indignation et être prêt à manifester cette indignation haut et fort collectivement et dans le quotidien.

Avec le temps, les expériences de nos employées et l'évolution du Centre, la signification du militantisme féministe a pris la forme suivante : individuellement, nous devons nous indigner pour dénoncer les paroles dénigrantes et les mythes envers les femmes, éduquer la population sur les enjeux auxquels les femmes font face, participer aux discussions dans les médias sociaux et autres, revendiquer des services en français et militer pour les droits des femmes, nous impliquer dans les activités communautaires de revendication, de sensibilisation et de prévention, faire du bénévolat auprès des organismes de femmes et vivre les principes féministes au quotidien. Ensuite, nous devons éduquer la communauté à en faire autant.

Nous nous sommes rendu compte qu'afin d'obtenir un certain succès à transmettre le message, il est primordial de tenir compte des réalités propres au milieu dans lequel nous œuvrons.

\section{Réalités rurales}

Le milieu rural est caractérisé par un contexte social et historique particulier. Dans notre région, nous avons constaté que les idéologies et valeurs religieuses conservatrices sont encore très présentes dans les communautés qui vivent un isolement géographique et social. Le tabou de la violence faite aux femmes, particulièrement l'agression à caractère sexuel, est toujours aussi présent dans nos communautés.

En milieu rural, il est aussi difficile de dire qu'on va faire une activité comme un « flash mob ", puisqu'il n'y a pas de lieu commun. Les endroits de rassemblement existent dans certains villages et, comme il y a plusieurs villages, nous n'arrivons jamais à rassembler tout le monde en même temps. Prenons, par exemple, les vigiles du 6 décembre : nous devons tenir trois cérémonies dans trois lieux différents afin de permettre à un plus grand nombre de personnes de participer. Comme il y a peu de personnel pour le faire, cela est très exigeant. La communauté se fie encore aux organismes pour organiser ce genre 
d'événement. Nous constatons que l'indignation collective n'est pas encore assez forte pour avoir un impact sur toute une communauté et amener celle-ci à s'organiser.

Le transport en commun est inexistant, malgré les grandes distances qui séparent les centres plus peuplés. Cet isolement empêche les rassemblements de la population. La communication, l'éducation et la prévention, qui se font en situation de rassemblements publics, sont presque inexistantes à cause de ces réalités. Et bien qu'elles soient de plus en plus rares, certaines communautés ont encore difficilement accès à Internet.

Aussi, les femmes, celles qui ont une cause à défendre en lien avec la violence qu'elles subissent, sont encore influencées par un patriarcat plus prononcé. Oseront-elles organiser une activité de revendication ou de militantisme? À quel prix? La population les supportera-t-elle? Cela est toujours facile quand il s'agit d'enfants malades ou de commerces qui ferment, mais quand on parle de violence faite aux femmes, l'enjeu est trop grand. Nous sommes confrontées à de nombreuses personnes qui pensent que cela n'existe pas dans leur communauté. Le manque d'anonymat présente aussi un obstacle. Si une femme s'associe à un événement en lien avec la violence et la revendication de ses droits en tant que femme, va-t-on la juger?

Le fait que les organismes communautaires qui parviennent à s'ancrer dans les communautés rurales ne vivent souvent que de projet en projet, il est difficile d'établir un lien de confiance avec la communauté et de maintenir une constance de services : personnel sous-payé, roulement de personnel, démarrage de projets qui ne peuvent être menés à terme à cause de l'absence de financement continu, difficulté des bailleurs de fonds à reconnaître les spécificités francophones et rurales. Alors qu’en milieu urbain, il est peut-être un peu plus facile d'aller chercher des commanditaires. Au niveau rural, ce sont toujours les mêmes petites entreprises, peu nombreuses, qui sont sollicitées pour toutes les causes.

Comme dans la société en général, nous retrouvons une population croissante de personnes plus âgées et moins prêtes à changer leur façon de penser. Quand une personne est socialisée par le patriarcat et que celui-ci est encore bien présent dans une communauté rurale, il est difficile de changer les attitudes bien ancrées des personnes de 70 ou 80 ans.

\section{Impacts sur le mouvement}

Les éléments mentionnés ci-dessus et l'émancipation du cadre religieux et conservateur, l'évolution plus lente des discussions et le patriarcat encore très agrippé, en plus de la 
lutte pour des services en français, font que la lutte des femmes avance à un rythme plutôt lent. La mobilisation communautaire est difficile aussi entre organismes, et les bailleurs de fonds gouvernementaux travaillent en silo et font en sorte que les organismes sont souvent en compétition l'un contre l'autre, toute l'énergie étant mise à sécuriser la survie de l'organisme. Les pratiques de développement communautaire demandent encore beaucoup d'énergie et de collaboration et ne sont pas toujours faciles à mettre en place. Souvent, l'engagement d'un organisme à faire du militantisme va dépendre de sa direction et, si celle-ci change, tout peut être à recommencer.

Dans une ère où il est souvent dit que l'égalité entre les genres est atteinte, les preuves que ce n'est pas tout à fait exact sont bien présentes. Le fait que nous devions encore faire des pieds et des mains pour avoir la permission de présenter des ateliers de prévention de la violence faite aux femmes dans certaines écoles catholiques primaires et secondaires, le fait qu'une victime d'agression à caractère sexuel soit encore blâmée pour l'agression ou le fait que les femmes qui portent plainte à la police à la suite d'une agression ne soient pas crues sont des preuves flagrantes de cette inexactitude. Et bien que ce genre de situation se trouve dans bien des milieux, soit urbain, suburbain, francophone, anglophone, autochtone et autres, sa prépondérance est plus flagrante dans les milieux où les occasions de discussion, d'éducation et d'échange sont beaucoup plus difficiles.

Dans les communautés rurales ou éloignées, les militantes et les organismes militants doivent partir d'une base plus simple et travailler plus fort pour créer des occasions de conversation au sein de leur population. Le cheminement et l'évolution de la conversation suivent un rythme bien différent de ce qui se passe en milieu urbain. Dans les zones urbaines, il y a plus d'organismes qui promeuvent le même message, et plusieurs spécificités peuvent être prises en considération. Par exemple, avec une plus grande proportion de femmes autochtones, ces femmes arrivent à s'organiser des services qui répondent à leurs besoins. Dans un milieu rural où moins de $1 \%$ de la population s'identifie comme autochtone, les chances que ces femmes se retrouvent et s'organisent sont bien minces! Donc, qui revendiquera, éduquera ou soutiendra ce militantisme?

\section{Leçons apprises et réussites}

Depuis dix ans déjà, le Centre Novas tente de s’ancrer dans la communauté de PrescottRussell. Plusieurs essais ont été faits afin de changer les attitudes. Notre façon de faire les choses a évolué, et nous en sommes venues à considérer que, pour amorcer le changement, nous devons d'abord faire de la sensibilisation et de l'éducation. Selon nos expériences, 
des gens mieux informés sont plus aptes à ouvrir leur esprit au changement d'attitude. Nous devons aussi respecter le rythme de la communauté. Nous avons choisi de nous allier à des regroupements locaux et provinciaux afin d'avoir accès à des ressources et à des partenaires plus nombreux. Sur le plan local, la Coalition de Prescott-Russell pour éliminer la violence faite aux femmes nous permet de travailler en collaboration avec les autres organismes de la région. Au niveau provincial, l'organisation Action ontarienne contre la violence faite aux femmes nous permet d'être branchées sur les activités et les ressources à travers la province. Les efforts et les partenariats sont beaucoup plus efficaces quand nous travaillons avec d'autres organismes expérimentés et ayant des ressources humaines et financières à partager!

Nous organisons aussi des activités communautaires qui nous donnent une visibilité, une place publique pour dénoncer, nous indigner. Et nous nous assurons de solliciter nos partenaires communautaires et d'affaires afin de passer le mot. Nous faisons le tour de la région pour présenter nos activités et ainsi permettre à un plus grand nombre de citoyennes et citoyens de participer.

Par exemple, la marche des hommes « un mille en talons hauts " ou Walk a Mile in Her Shoes ${ }^{\circledR}$ a été créée en 2001 par Frank Baird, aux États-Unis. Le but était de sensibiliser les hommes, de façon humoristique, à la réalité de la violence sexuelle faite aux femmes. Un petit groupe d'hommes, qui a " osé » faire une marche en souliers à talons hauts, a réussi à créer un mouvement mondial qui recueille, chaque année, des milliers de dollars pour les centres d'aide et de lutte contre les agressions sexuelles, les maisons d'hébergement pour femmes victimes de violence conjugale et autres programmes de prévention des agressions à caractère sexuel. Dans Prescott-Russell, nous avons mis sur pied un comité organisateur d'hommes alliés à notre cause. L'activité se tient en mai, durant le mois de sensibilisation aux agressions à caractère sexuel, et se veut aussi une collecte de fonds pour le programme de prévention/sensibilisation du Centre Novas. En mai 2017, nous tenions notre $9^{\mathrm{e}}$ édition annuelle de la marche et avons eu plus de 75 marcheurs et reçu quelque 3000 en dons! Souvenons-nous que les distances qui séparent nos communautés nous obligent à réduire nos attentes face au nombre de participantes et participants et de donatrices et donateurs à nos activités. Ce comité proactif a accepté de participer à une nouvelle campagne de sensibilisation par et pour les hommes. Des affiches arborant les hommes membres du comité et leurs réflexions sur la violence faite aux femmes seront distribuées auprès des commerçants locaux (restaurants, garage de mécanique, entreprises d'équipement agricole, etc.) afin de passer le message de façon « discrète » et de joindre les hommes dans leurs milieux. 
Le Centre Novas, en collaboration avec son regroupement local, soit la Coalition de Prescott-Russell pour éliminer la violence faite aux femmes, tient annuellement la marche La rue, la nuit, femmes sans peur. Ce moment de revendication des femmes au droit de circuler en liberté, sans toujours craindre de se faire agresser, fait le tour de la région, et nous marchons avec une centaine de femmes depuis déjà dix ans.

Enfin, nous nous employons activement à former la relève avec tous nos outils et notre connaissance, que ce soit en présentant nos ateliers de prévention aux jeunes dans les écoles, en prenant des stagiaires dans notre milieu de travail ou en recrutant pour le conseil d'administration. Nous ciblons les jeunes d'ici, ceux qui sont les décideuses et décideurs de demain.

\section{Conclusion}

Le militantisme chez nous, c'est encore de trouver des façons de briser le silence, les tabous et les mythes existants en créant des occasions pour la discussion et la réflexion. Linformation est le pouvoir de changer des attitudes.

Le Centre Novas continue de chercher les moyens les plus adaptés aux réalités de sa communauté rurale et minoritaire francophone pour militer. Ce que nous avons appris de nos essais et erreurs est la nécessité de commencer à la base et de respecter le rythme; cela nous permet d'ajuster nos objectifs et d'en arriver à un changement progressif d'attitude. La connaissance des réalités de notre communauté, plus particulièrement, nous permet d'adapter nos pratiques et notre militantisme. Maintenant, nous devons réussir à faire comprendre ces ajustements à nos bailleurs de fonds afin de pouvoir continuer notre travail. Un jour, peut-être pas trop lointain, la communauté fera-t-elle, elle-même, le militantisme nécessaire pour changer ses attitudes!

Comme le conclut si bien l'auteure du Manuel de résistance féministe :

Nos moyens de lutte et nos stratégies d'action sont diversifiés, à l'image de celles et de ceux qui composent les mouvements féministes, ici comme ailleurs. De la prise de parole à l'action collective, de l'éducation populaire à la désobéissance civile : continuons de faire entendre nos voix! Affirmons haut et fort notre féminisme. Continuons de nous indigner et de lutter contre toutes les formes d'oppressions, de nous réapproprier et de transmettre l'histoire de nos luttes, de renforcer nos alliances et nos solidarités, de célébrer nos victoires. 
Et surtout, demeurons convaincues que nous avons le pouvoir de changer le monde! (Surprenant, 2015, p. 162)

\section{Notes}

1 Bien que ces données datent du moment de rédaction du Rapport de l'étude de besoins sur les services à développer pour les victimes et survivantes francophones d'agression à caractère sexuel dans Prescott-Russell (2005), elles demeurent les mêmes à ce jour.

2 Pour obtenir tous les détails sur les assises du Centre Novas, voir le plan stratégique 2014-2019 rédigé par Convergence (2014).

\section{Bibliographie}

COLLECTIF (2009). Le Petit Larousse illustré 2010, Éditions Larousse, 1881 p.

COMITÉ ENCADREUR DU PROJET NOVAS (2005). Rapport de l'étude de besoins sur les services à développer pour les victimes et survivantes francophones d'agression à caractère sexuel dans PrescottRussell, Casselman, 43 p.

CONVERGENCE (2014). Planification stratégique 2014-2019 du Centre Novas-CALACS francophone de Prescott-Russell, Rapport final, Casselman, 24 p.

SURPRENANT, Marie-Eve (2015). Manuel de résistance féministe, Éditions du remue-ménage, 182 p.

WALK A MILE IN HER SHOES® (2017). The International Men's March to Stop Rape, Sexual Assault \& Gender Violence, réf. du 14 août 2017, http://walkamileinhershoes.org 